\title{
(Co-)Constructing a theory of mind: From language or through language?
}

\author{
Hande $\operatorname{llgaz}^{1}$ (D) Jedediah W. P. Allen ${ }^{1}$
}

Received: 19 July 2019 / Accepted: 12 February 2020

(c) Springer Nature B.V. 2020

\begin{abstract}
There is a large body of empirical work that has investigated the relationship between parents' child-directed speech and their children's Theory of Mind development. That such a relationship should exist is well motivated from both Theory Theory and SocioCultural (SC) perspectives. Despite this general convergence, we argue that theoretical differences between the two perspectives suggests nuanced differences in the expected outcomes of the empirical work. Further, the different ontological commitments of the two approaches have (mis)guided the design, coding, and analysis of existing research and imply different future directions. We discuss five areas of extant research that can be extended and diversified most coherently by adopting a SC framework.
\end{abstract}

Keywords Theory of mind · Folk psychology $\cdot$ Socio-cultural theory · Theory theory $\cdot$ Mental state talk

We seek to examine the role that social experience plays in the development of children's ability to reason about the mental perspectives of self and others. This ability, from here on referred to as theory of mind (ToM), is widely recognized as a sociocognitive ability that benefits from children's experience of perspectivally rich talk in meaningful social interaction contexts (e.g., conversations, storytelling, pretend play). One indicator of perspectivally rich talk is the use of mental state terms that describe mental processes that are not directly observable. Two approaches (i.e., theory theory and socio-cultural theory) underscore the importance of child-directed mental state language in theory of mind development. While evidence for the relationship between the richness of child-directed mental state talk and theory of mind has been taken to

\footnotetext{
$凶 \quad$ Hande Ilgaz

hande.ilgaz@bilkent.edu.tr

Jedediah W. P. Allen

jallen@bilkent.edu.tr

1 Department of Psychology, Bilkent University, 06800 Ankara, Turkey
} 
support either framework, we argue that there are important nuances in the expectations of each for the relationship between ToM and child-directed mental state talk.

In essence, we argue that the Socio-Cultural (SC) framework takes children's social interactional experience (including linguistic interaction) to be constitutive of the ability to reason about mental perspectives. For this approach, children's ability to reason about perspectives from a third-person vantage point emerges in the context of secondperson social interaction and is not present a priori. In contrast to this approach, the Theory Theory (TT) position holds that the child is equipped with a motivation to build theories that capitalizes on affirmed associations between behavior and hypothesized mental states. Further, the child understands others through taking a third-person perspective that is at theoretical remove from the "rough ground" of real-time social interaction (Reddy 2007). Children's linguistic experience in social interactions serves an instrumental purpose, a platform upon which children gather data in order to construct and revise their theories of mind.

We argue that the contrast in the explanatory power of these two approaches lays in deeply rooted differences in their background frameworks for how ToM emerges and the mechanism(s) by which it develops. It is our contention that the extant research provides relatively indiscriminate support for either theory. This is largely due to methodological approaches that have taken child-directed talk as a constant rather than a dynamic interactive variable. Specifically, the aspects of talk that would be affected by interactional variables have been held constant which has eliminated the possibility of examining how child-directed talk and its effects change as a function of context, conversation partner, and characteristics of the child (e.g., level of knowledge). In addition, we will propose that the ways in which mental state language has been coded has narrowed its scope over time to focus on a select set of mental state clusters that are used from a third-person perspective (i.e., to describe story characters' mental states and processes). However, there are two levels of perspective available to a reader and a listener: (1) reflecting on and inquiring about the readers' or listeners' mental states/processes in reaction to the story (i.e., second-person perspective); and, (2) reflecting on the characters' mental states/processes (i.e., third-person perspective). We will argue that even for research that explicitly claims to investigate the issue from a socio-cultural perspective, their methods and design have left them unable to pursue research questions within such an approach.

The particular focus of this paper is to examine the different expectations that TT and SC approaches hold for the relevance of child-directed language for theory of mind development. We aim to emphasize the importance of contextual variables that we propose would allow for a clearer differentiation between the two approaches. To that end, we will first briefly discuss each theory's underlying ontology for the development of theory of mind. We follow this with a brief discussion of how each approach construes the role of child-directed speech in supporting children's theory of mind development. Lastly, we discuss five areas for the expansion or modification of research that should better enable an evidence-based differentiation of the two approaches. Throughout the paper, we will illustrate our arguments with empirical work that mostly relies on storytelling/picture book contexts as the majority of relevant research focuses on these sorts of interaction contexts. 


\section{Two contrasting ontologies}

Theory theory. The TT approach makes several explicit assumptions about the nature of development (Gopnik 1996; Gopnik and Wellman 1992; Wellman 1990). The core idea is that children's knowledge is structured into different domains and that those structures are theory-like. In turn, children are thought to be akin to little scientist who gather data to confirm or refute their existing theories. To get the whole process of "hypothesis testing" started, infants must be equipped with foundational theories that are innate. Accordingly, this version of TT is referred to as starting-state nativism (Gopnik 2003). Starting-state nativism differs from regular nativism in that the starting theories are open to qualitative revision. That is, children are able to construct new content for their theory revision processes such that development involves qualitative changes.

Two central difficulties arise for the TT framework espoused by Wellman and his colleagues: first, the theory revision process has no way to account for new conceptual content; ${ }^{1}$ second, culture can only be instrumentally related to development. Regarding the first difficulty, the inability to account for the development of content about mental states is what lead theory-theorists to adopt the starting-state nativist position in the first place. The issue is that the hypothesis generation process needs the content of the hypotheses to already exist in order for them to be generated. The problem for TT is that this constraint applies to the generation of new content later in development as much as it applies to the starting content in the foundation (Allen and Bickhard 2011). This point is what lead Fodor (1975) to his "mad-dog" nativist conclusion (i.e., that most lexical concepts had to be innate). More problematic is that Fodor's argument is a logical point that cannot be resolved by evolution any more than can it be resolved by development (Bickhard 1991). Thus, the function of the starting-state nativist position (i.e., to ground the theory generation process) does not resolve the difficulty of accounting for new representational content. ${ }^{2}$

The other difficulty for the TT position concerns its general relationship to culture. For TT, culture will contribute to the child's development through social interaction in general and through socio-linguistic interaction in particular. However, for TT, we argue that the relationship with culture is limited to an instrumental role. The basis for this claim lays in TT's characterization of the child as a little scientist. The child is tasked with the generation of new hypothesis that can accord with the data that is being accumulated through experience. Many of those experiences will be social but for the child-scientist it is all just more information or data to be analyzed from a third-person observer perspective (Gallagher 2001; Nelson 1996, 2007, 2009; Reddy 2007). For the TT approach, the social environment does not co-constitute the generation process for

\footnotetext{
1 While the theory revision process has been formalized through the use of new statistical modeling, the conceptual point regarding new content holds (i.e., to generate new hypothesis requires new content and nothing about Bayesian modeling will provide that new content).

2 For an extended discussion about the problem of foundationalism for developmental nativist and empiricist positions in general, see Allen and Bickhard 2013. For a more focused discussion on the problem of foundationalism for TT, see Mirski and Gut 2018.
} 
the testing of new hypotheses. ${ }^{3}$ Accordingly, culture's influence is limited to the timing of the constructive processes (Wellman and Liu 2004; Wellman 2014) but what gets constructed will be intrinsic to the theory generation processes of the child/species.

Socio-cultural approaches. Both the issue of emergence and of culture being able to play a constitutive role in development are resolved by SC approaches (Bruner 1990; Carpendale and Lewis 2006, 2014; Nelson 1996, 2005, 2007, 2009, 2010; Nelson et al. 1998, 2003; Tomasello 2019). SC approaches emphasize social interaction (and reaction) as the proper locus for the emergence of social understanding. The essential character of this approach is aligned with, and in some cases derivative from, the general framing of development by Vygotsky (1997; 1st publication 1931) in which all uniquely human cognitive functioning has its origins in the social sphere prior to an internalization process that eventuates in more individualized knowing. This focus on social interaction has a further consequence in terms of the relevant unit of analysis for empirical methodology. For TT, the unit of analysis is the child's mentalstate attribution activity while for a SC approach it will be the child's interaction in an already meaningful social situation. Lastly, the focus on social interaction situates these approaches within a generally pragmatist or action-based framework for studying mind and cognition more broadly. The best known version of an action-based approach is Piaget's (1954) but contemporary versions also exist (Bickhard 2009a, b). ${ }^{4}$

For action-based approaches in general and for socio-cultural approaches in particular, the constructive processes of the child are not generating hypotheses with conceptual content (at least not at first); instead, the constructive processes are generating affordance spaces of interactive potentialities. Children's foundational understanding of the physical and social world is an understanding of interactive possibility. In turn, if knowing/understanding is competent interaction with the world, then new interactive competences constitute the emergence of new knowing and the purported need for a foundationalism (i.e., starting-state nativism) is unnecessary (Allen and Bickhard 2013). Further, interactive potentialities are constituted by relationships between the child and the environment. While the interactive potentialities of the physical world are quite different from those of the social world, the relational aspect of

\footnotetext{
${ }^{3}$ What would it mean for the TT position to integrate the social environment into the constructive processes? Perhaps we could think of the child as co-constructing their hypotheses in order to give culture a more constitutive role. For example, co-constructing hypotheses could be similar to the co-construction of memories through the process of reminiscing. Perhaps the co-constructed hypotheses about the mind would be manifest through conversations about the actions of other people. Further, the pre-linguistic version of this would be the child's second-person interactions with other adults. Accordingly, TT could give culture a more constitutive role by focusing on second-person interactions in infancy; the integration of rudimentary language would be seen as increasing the complexity of those second-person interactions in toddlerhood; and, finally, more complex language would be understood as helping the preschooler to reflect on and take a third-person perspective toward themselves and others. However, this would seem to result in TT giving culture a more constitutive role by aligning itself with a socio-cultural approach. Further, the underlying issues related to emergence would remain.

${ }^{4}$ See embodied, enactivist, ecological, and phenomenological approaches to cognitive science for different degrees of convergence with action-based approaches in general and socio-culture approaches in particular (Chemero 2009; Gallagher 2001; Hutto and Myin 2012; Ziemke 2003). That said, enactivist, ecological, and phenomenological approaches differ from action-based approaches in that they tend to reject the possibility of modeling representation altogether; and, embodied approaches that do not share the anti-representationalism of the other three tend to be overly reliant on information-processing assumptions in which the "body" still has no necessary/constitutive connection to cognition.
} 
all knowing means that the social environment is co-constituting the interaction and thereby co-constituting the knowing. In this way, an action-based approach opens the way for culture to play a constitutive role in development (Christopher and Bickhard 2007). In short, the action-bases of socio-cultural approaches resolve the difficulties concerning emergence and opens the way for the socio-cultural environment to coconstitute development.

\section{The effect of child-directed language on ToM development}

Theory theory and socio-cultural theory share commonalities that render it possible to use the results of empirical work on the effects of language for ToM development to support either approach. ${ }^{5}$ Both theories posit that children who interact with others through language in meaningful contexts (e.g., play, reminiscing, storytelling) will show better ToM performances (Wellman 2014; Nelson 1996, 2007). A plethora of research indeed shows that this is the case (e.g., Devine and Hughes 2018; Tompkins et al. 2018). In contrast, children who are deprived of such perspectivally rich talk will end up with poorer ToM abilities, as research with congenitally deaf children born to hearing parents also evidences (Peterson and Siegal 2000). In broad strokes, all research that documents a relation between children's ToM abilities and the quantity of the perspectival language they hear can be taken as supporting either framework. What may differentiate these two theories will depend on work that makes specific predictions based on their conceptualization of the emergence and development of ToM.

Wellman and his colleagues have provided a robust theoretical and empirical research program that was intended to elucidate the development of children's changing theories of mind (for a comprehensive review, see Wellman 2014). From the TT perspective, children are tasked with the challenge of understanding why people behave the way they do and must construct theories based on available data (this is in contrast to the SC perspective in which children are tasked with the challenge of making meaning through coordinated interaction). The TT approach pays homage to Piagetian views of the child as an active constructor of knowledge. Also in the Piagetian tradition, Wellman sought to explain what was universal in children's changing ToM abilities throughout early development. Creation of the ToM scale (Wellman and Liu 2004) was one important outcome of these efforts, in that different steps in the scale were argued to provide the conceptual precursors for the development of

\footnotetext{
5 One difficulty for the social-cognition literature in general is that the empirical work seems to be accommodated by all of the different approaches (Miller 2016) — this includes simulation theory and modularity theory. However, if there are to be meaningful differences between the approaches then different empirical work should fit better for some approaches than for others. Further, it is clear that the sorts of empirical research programs pursued by researchers differ in terms of their background frameworks. For example, the modularity approach has limited empirical research programs or they have tended to focus on children with developmental disorders. In contrast, the constructivism of TT and SC approaches have resulted in robust empirical research programs that tend to focus on (linguistic) social interaction at different developmental periods. Our goal in the current study will be to illustrate some of the particulars of empirical research that we argue are better motivated and interpreted from within the socio-cultural tradition over the TT perspective.
} 
new concepts. The relative invariance of the sequence across cultures (e.g., Shahaeian et al. 2011; Wellman et al. 2006) suggested some universal constraints on the child's constructive processes themselves. The last similarity with $\mathrm{Piaget}^{6}$ would seem to be in terms of the secondary role for language. For TT, language exposure is more fuel for the theory revision processes, but the new mental-state concepts are not created by such exposure.

In contrast to the relative unity of TT in terms of theoretical foundations and empirical research program, the SC framework is more diverse. Amongst the most well-known advocates of versions of a SC approach are Jeremy Carpendale and Charlie Lewis, Katherine Nelson, and Michael Tomasello. These scholars can all be construed to have adopted a socio-cultural approach for several reasons. First, they all emphasize that children are active in the process of creating social understanding. This is a point they would share with the TT position. Second, and in contrast to the TT view, this approach takes the social construction of understanding as essential. In this view meaning making through language is already situated in a meaningful social context where the child not only constructs a culturally mediated understanding but is continually engaged in a social process of the co-construction of meaning in interaction with others. This is essentially a co-constructivist account of development that has to varying degrees been influenced by Vygotsky (Fernyhough 2008; Nelson 1996; Tomasello 2019). Third, this approach emphasizes the constitutive role of social interaction in general, and linguistic interaction in particular for the development of social understanding. Language development is co-constitutive of social meaning for the child, it is not a mapping between pre-existing contents and their linguistic labels; instead it is an elaboration of the ways in which meaning can be constructed. For this approach, the development of social understanding and of language are not at theoretical remove from the "rough-ground" of social engagement. Accordingly, the relative decontextualization of language is understood as a developmental outcome, not as a starting point.

According to two of the progenitors of a socio-cultural approach (Bruner 1990; Nelson 1996), for the development of children's folk psychological understanding, meaning making tends to capture the idea of language mediated understanding of social situations. However, for such approaches, any actual language is considered cultural in the sense that it cannot itself be abstracted away from the historical and cultural embeddedness of its use. One methodological consequence of this is that the focus on language by SC theorists is typically in terms of the sorts of narrative practices to which children are participants. That is, these practices are themselves a manifestation of the culture, both in terms of the function(s) and style of the practice (for an application of these ideas involving mother-child reminiscing see, e.g., Fivush 2019; Melzi et al. 2011; Miller et al. 2001).

Importantly, a language mediated folk-psychological understanding of social situations is not the only way to make (social) meaning. Other prominent proponents of a socio-cultural approach focus on pre-linguistic forms of making meaning that serve as a necessary starting point for the learning, and incorporation, of language into the

\footnotetext{
6 What Wellman does not share with Piaget is the underlying action-basis to cognition and mind more broadly. Consequently, he does not share with SC approaches in their focus on the pragmatics of (linguistic) social interaction.
} 
meaning making process. ${ }^{7}$ For a well-studied example, consider social activity involving pointing (Carpendale and Carpendale 2010; Tomasello et al. 2007). Meaningful pointing seems to develop starting at around 10 months of age and infants can use pointing for multiple different communicative functions (e.g., for imperative, declarative, or informative purposes). Tomasello argues that this sort of meaning making is possible because infants are already capable of attributing the mental state of intention to others (Tomasello 1999). In contrast, Carpendale and Lewis (2006) propose their own version of an action-based approach as the ultimate origins of meaningful social understanding.

Explicitly adopting an action foundation helps make sense of the concept of meaning making in ways that are broader than just language interaction. In the realm of the social, meaning is still interactive/action-based at its core. Children must come to learn about how to interactively characterize different sorts of social situations. This can be for things as mundane as diaper changes or as "complex" as waving goodbye to guests. From this perspective, the problem for constructing social ontology is the need for common ground between the agents attempting to interactively characterize a situation (Bickhard 2008). That is, another person's interactive characterization of a situation is, mostly, not transparent (i.e. perceptually available). Accordingly, for us to jointly characterize the situation with sufficient overlap in ways that enable successful interaction requires a common understanding of the situation. In turn, common ground is established by understanding the type of situation that is involved. Sometimes the type of situation is learned jointly through an interactive history of variation and selection (e.g., the diaper changing). Other times, children must come to learn about culturally defined situations through processes of imitation (e.g., waving goodbye to a guest). From this perspective, social meaning is fundamentally constituted by these social situations - the mutually held interactive characterizations of the situation-and the incorporation of language into this process allows for more complex interactive characterizations (Bickhard 1980).

In sum, given the focus of the current manuscript on mother-child discourse, the Bruner/Nelson interpretation of meaning making will be useful for most of the discussions; however, our understanding of meaning making is broader than how these stalwarts tended to use the concept. In particular, our strong commitment to an actionbased foundation means that the child can make "folk-psychological" meaning before and after developing language.

Misinterpretations of SC approaches from developmental psychology tend to characterize the position as suggesting that the child is a passive recipient of adult instruction. For instance, Wellman (2014) contends that according to the socio-cultural perspective "Theory of mind arises from the sheer accumulation of information from others. Adults tell and show children how to think about (and talk about and interact with) people as mental beings, and children go from being ignorant about all these to absorbing the ideas of those around them" (p. 7). This misconstrual strips socio-

\footnotetext{
${ }^{7}$ For a socio-cultural approach, including that of Katherine Nelson, language is not generally considered the starting point of making (social) meaning. Instead, language builds on children's existing understanding of the meaningfulness of different types of social situations. In this way, socio-cultural theorists do not tend to see language as its own domain of development; instead, language development is an emergent outcome of the social-cognitive domain.
} 
cognitive development of the critically constitutive effect of social interaction, and thus, of culture. Such a view, reduces culture to a bundle of information to be taught to novices, as opposed to a critical constituent of socio-cognitive development which can be used in the process of reflection via folk-psychologies (Bruner 1990). Lastly, such a characterization renders language development as a vehicle for the transmission of conceptual content from expert adults to novice children.

In our view, the difference between SC and TT theories is not whether they adhere to a "child-as-active" form of constructivism. It is clear that both approaches assign an active role to the child in constructing socio-cognitive understanding. The divergence is whether the child engages in co-construction and whether the co-construction is focused on the pragmatics of successful social interaction or the development of new theories about other's minds. ${ }^{8}$ For TT, the child constructs theories based on his interactions with other social agents. However, in these social interactions the child seems to be tasked with extracting the human universal (i.e., ToM) that is embedded in the cultural diversity (folk psychologies). In turn, cultural influences are restricted to the timing of milestones and to small changes in a relatively invariant developmental sequence. Work by scholars who take the TT approach has shown differences in the age of achievement for false belief understanding by culture. Most notably, it has been shown that children from mainland China and US are similar in timing of FB achievement as compared to children from Hong Kong who show success in FB tasks later (Liu et al. 2008). In addition, it is now a well-documented finding that children from China, Iran, and Turkey master knowledge access tasks before diverse belief tasks, this is the reverse sequence when compared to American and Australian children (Selcuk et al. 2018; Shahaeian et al. 2011; Wellman et al. 2006). TT acknowledges that folk psychologies, parenting practices, and societal expectations are the likely reasons for such differences. However, whether folk psychologies themselves have meaningful effects on children's conceptions of mental states has not been considered rigorously. In other words, whether the meaning of the belief concept is different for individuals of different cultures has not been investigated (Lillard 1998). ${ }^{9}$

Extant research from a TT perspective acknowledges that cultural variability exists when children are administered carefully translated versions of the same tasks with a few culture-conscious adjustments. These adjustments have included substituting objects in scenarios with more familiar ones (e.g., Shahaeian et al. 2011), or the word "to think" with the pragmatically more appropriate Chinese verb "to falsely think' (Wellman et al. 2006). However, TT does not regard culture as co-creating development and so cultural variability is thought to push around the development of the children's theories of mind without changing their constitution. As stated previously, the finding that the frequency of child-directed mental state language affect children's theory of

\footnotetext{
8 The underlying reason for this divergence is in terms of what challenge social understanding is supposed to overcome (Carpendale and Lewis 2014). For TT, the challenge is to understand other's behavior by understanding their minds. For most SC approaches, the challenge is to make meaning of the social situation through coordinated social engagement.

9 Lillard has provided an excellent survey of some of the cultural variation for beliefs about others' minds and behavior (i.e., folk psychologies). Wellman acknowledges this variation but argues that it does not undermine the quest for that which is universal. We hope that our explication of SC approaches can further open the door to the essential role of such cultural diversity while maintaining the quest for that which is universal.
} 
mind abilities supports either position. However, the socio-cultural approach should make specific predictions, which if documented, would not be explained as cogently by the TT approach.

In this paper, we aim to explicate the ways in which research on child-directed speech should be expanded to allow for disentanglement of which of these frameworks may best explain the development of ToM abilities. Specifically, we will discuss five areas in which the extant work can and in our opinion should be extended and diversified: (i) the interaction contexts in which language is elicited, (ii) the scope of mental state language that is coded, (iii) the choice of perspective from which the mental state talk is coded (esp. in storytelling: character vs. adult and child), (iv), whether children benefit from the same aspects of child-directed mental state talk with all conversation partners (e.g., the mother vs. the father, vs. older or younger siblings), and finally (v) whether mental state language taken as a dynamic scaffolding variable shows variability according to both context and conversation partners.

The relation between child-directed talk and ToM has been the focus of two recent meta-analysis (see Devine and Hughes 2018; Tompkins et al. 2018). Some of the points we discuss have also been elaborated in these meta-analytic reviews as future research directions. Our aim here is not to go into the details of the findings of each study but to provide commentary on a list of issues within the extant research. We believe that this undertaking may serve to highlight the theoretical importance of some of the issues that we discuss by drawing attention to aspects of research design, coding, and analysis.

\section{The effect of social interaction context}

Both TT and SC approaches propose that child-directed talk in meaningful interaction contexts support children's theory of mind development. From the TT perspective, the contexts themselves don't matter so long as they provide children with data relevant for building theories about mental processes. In contrast, for the SC approach the contexts provide children with different types of interaction possibilities that are used to develop an understanding and coordination of others' perspectives. In this way, the context is itself intrinsic to the development of social-cognitive understanding. ${ }^{10}$

At this point, a sizable body of research has investigated the relationships between child-directed talk and children's ToM abilities in interactions that use wordless storybooks (e.g., Adrián et al. 2005, 2007; Racine et al. 2007; Slaughter et al. 2007), commercial storybooks with text (e.g., Ensor et al. 2014; Symons et al. 2005; Tompkins 2015), picture stimuli (LaBounty et al. 2008; Ruffman et al. 2002; Taumoepau and Ruffman 2006), play (Meins et al. 2002, 2013; Symons et al. 2006), and reminiscing (e.g., Laible 2004, 2011; Ontai and Thompson 2008). In addition, a few studies have used a self-report measures in which parents are asked to report their preference for mental state content in everyday situations in which they might interact with their children (e.g., Peterson and Slaughter 2003).

\footnotetext{
10 With their eventual mastery of ToM children will develop a relatively decontextualized understanding of others (i.e., a decontextualized understanding is a developmental outcome).
} 
The recent meta-analysis by Devine and Hughes (2018) included all international studies that were written in English. This analysis did not show a differential effect of context (storybook/picture discourse vs. play/conversations vs. self-report) for the relation between parental mental state language and children's ToM abilities. In contrast, Tompkins and colleagues' meta-analysis (2018) which included English-speaking children only but also included reminiscing as a context showed that parental mental state talk in storybook/picture discourse, self-report, and play contexts showed significant relations to children's ToM, but other conversations in naturalistic settings (e.g., meal time) and reminiscing did not.

These results provide evidence for the relation between child-directed mental state talk in perspectivally rich contexts and children's ToM development both crosssectionally and longitudinally (Devine and Hughes 2018). This is a position supported by both the TT and SC perspectives (Carpendale and Lewis 2006; Nelson 2007; Wellman 2014). However, these results cannot inform us about whether the support of mental state discourse varies by context for the same group of children. Almost all of the studies included in the meta-analysis have included a single context (i.e., they did not manipulate context within or between groups of children). The meta-analysis is then combining data from multiple studies in one context (e.g., storytelling) and comparing these to the combination of studies that have collected data in a different context (e.g., play). In addition, context and age effects are obscured by the fact that the first meta-analysis (i.e., Devine and Hughes 2018) has not been able to include age as a separate variable, but instead has included all children from toddlerhood to the kindergarten years. This age window includes children who are yet to pass FB tasks, those who are transitioning, as well as children who show consistently successful performance in FB assessments. The other meta-analysis (i.e., Tompkins et al. 2018) has included age as a separate variable but has also reported that the different contexts have not been studied for the different age groups. Generally, studies with infants and toddlers have used naturalistic conversation observations and play, whereas studies with older children have used storybook/picture and reminiscing contexts.

From a SC perspective, parents co-constitute the interaction with their children. They are not only sensitive to their children's developmental level but also the context in which the discourse occurs. To give an example, parents when telling a story from a picture book may make extensive use of these pictures through pointing and perceptual mental state words. The same parents may rely on perception words less when they are reminiscing about a shared event. Instead, the reminiscing context may elicit certain types of cognitive words (e.g., remember, forget). It is possible that which mental state talk subcategories relate to ToM performance depends on the context in which the talk is elicited.

\section{The scope of mental state discourse}

Early research concerning mental state language investigated the onset of children's mental state language productions to see when young children started labeling their own and others' emotions, desires, and beliefs (Bretherton and Beeghly 1982; Bretherton et al. 1981; Shatz et al. 1983; Bartsch and Wellman 1995). Bretherton and 
colleagues $(1981,1982)$ created a comprehensive coding scheme that included a diverse set of categories: perceptual (e.g., to see, to look), physiological (e.g., hungry, sleepy), affective (e.g., happy, sad), and cognitive (e.g. remember, forget) words as well as expressions that mark volition/ability (e.g., want, need, can), moral judgements (e.g., naughty, good), and obligation (should, have to). In contrast to this diversity, other researchers have elected to use a more limited coding scheme that focuses on children's expressions of desire and belief. Furthermore, belief words have been limited to a select number of key words and their variations (e.g., think, know, but not remember or forget; Shatz et al. 1983; Bartsch and Wellman 1995).

Separate from children's production, research looking at parent's child-directed talk have used coding schemes with differing levels of inclusiveness. Those with more inclusive categories have focused on: perceptual, desire, affective and cognitive words (e.g., Adrián et al. 2005; Slaughter et al. 2007); but sometimes excluding the category of perceptual words (LaBounty et al. 2008; Ensor et al. 2014). Some studies have coded and analyzed the frequency of "think" and "know" separately from other cognitive words. Finally, several studies have focused only on cognitive mental state talk that either differentiate "think" and "know" from other cognitive words (Adrián et al. 2007) or include all cognitive words in one category (e.g., Tompkins 2015). In addition to this diversity in coding schemes, some studies that have coded for different categories have opted in the analysis to use aggregate frequencies such that they did not report analyses of the mental state categories separately (Symons et al. 2005).

The meta-analyses with child-directed talk (Devine and Hughes 2018; Tompkins et al. 2018) confirm the findings reported in individual studies: total mental state language is related to children's ToM understanding. However, given the diversity in coding, it is pertinent to ask whether sub-categories of mental state talk are differentially related to this ability. In their meta-analysis, Tompkins and colleagues (2018) investigated whether any of the specific mental state categories (i.e., desire, affective, cognitive) was a more potent predictor of children's ToM understanding. Their results showed that cognitive mental state talk was a significant predictor of children's first-order ToM as compared to desire and emotion words. It is important to emphasize that the list of cognitive words that have been included in coding schemes is different across studies (i.e., select number of cognitive expressions vs. all cognitive expressions), and studies have used mental state coding schemes with different levels of inclusiveness (e.g., in general, there has been an unexplained lack of perception words). Consequently, it may be premature to conclude that ToM understanding is related, exclusively, to the frequency of cognitive words in parent's child-directed speech.

Another point of concern is the influence of culture as expressed through language. The studies included in the Tompkins et al. (2018) meta-analysis were exclusively with English-speaking samples. It is true that all languages have various lexical items in their vocabulary to demarcate mental processes. However, they also contain 'nonmental state' expressions to indicate such processes. One example is the extensive use of modal adverbials that do not neatly fall into the cognitive category. These express an attitude towards information on the part of the speaker that may serve to express probability, certainty or inference under the right pragmatic context (e.g., "He must not have studied for the exam given his performance"). Some studies have coded for 
these modal adverbs under the label 'modulation of assertion' (Ruffman et al. 2002; Taumoepeau and Ruffman 2006, 2008), however for most research, either it is not clear that these categories of mental expression have been considered, or it is not clear as to whether these expressions have been included in the cognitive word categories.

Another example that could elucidate cross-cultural differences can be observed in the case of Turkish which is an agglutinative language that makes extensive use of morpho-syntactic units (e.g., suffixes). For instance, in Turkish, speakers can express desires via utilizing suffixes that modify verbs to demarcate the modality for volitional wishes to express desires (i.e., -e, -a), and modality for volitional suggestions to express intention (i.e., -se, -sa). In addition, adverbs such as "bana/sana göre" (in my/your perspective) or modal adverbials "ben-ce/sen-ce" (according to me/you) are everyday linguistic expressions that children hear frequently to describe perspectival information. The utterance "Bence kaybolduk" (rough translation: According to me, we are lost), would express a belief without resorting to the use of proper mental state words. These uses constitute quick and easy linguistic shortcuts to express thoughts and beliefs that speakers mark with perspectival intent (Göksel and Kerslake 2005). These examples illustrate the importance of coding for perspectival content that includes but is not limited to mental state words, especially with non-English speaking samples.

Why should we care whether mental state language coding schemes include nonmental state talk categories that serves to express mental content in accordance with the pragmatic context and speakers' attitude towards knowledge? The most straightforward answer is that if our theory is not a passive empiricism, where the labels for mental state language (e.g., think and know) will suffice to communicate the concepts that underlie them (e.g., belief), then we should pay attention to all of the ways in which language is used to communicate about mental processes. In order to understand the dynamics of the language-ToM interaction, the literature is in need of longitudinal research that adopts a comprehensive coding scheme that takes mental state labels as one category, amongst several, of perspectival talk. Importantly, such research should not be limited to English-speaking samples. Such a research agenda would serve to support our understanding of the development of meaning making in diverse cultures so as to contribute to a more complete picture of our understanding the universal versus culture-bound folk psychological aspects of children's meaning making endeavors.

\section{The effect of referent}

Mental state talk serves to explicate the mental processes and mental attitudes of speakers in interaction with one another. Conversations, storytelling, forms of social play, and reminiscing are significant interaction contexts where children hear and use such language. Among these contexts, storytelling and sociodramatic play have a special place in that speakers establish common ground to talk about third-persons' mental processes. For instance, storytelling provides the adult-child dyad an arena to discuss the mental reasons behind a story character's actions (e.g., "The frog ran away from home because he missed his friends"). Similarly, in sociodramatic play children communicate about the desires, emotions, and thoughts of the character roles that they 
and their partner take. However, research that investigates this relation in play contexts is quite limited in comparison to research with the storytelling context.

The TT and SC approaches agree on the importance of children's experience in such contexts. For the TT approach the child gets to apply their fledgling theories of persons and learn from mismatches, whereas the SC approaches view these contexts as critical for children's entry into the way their community construes of and talks about minds. Research that focuses on the storytelling context has generally coded for parents' mental state talk in reference to story characters (e.g., Slaughter et al. 2007). This practice is in line with the assumption, that the value of storytelling is in the practice it provides in thinking about others' mental states. Parents' mental references to self (e.g., I wonder where the frog is hiding) or references to their child (e.g., where do you think the frog is?) have been eliminated from such research. This practice seems to be a misguided attempt to code for only the genuine mental state language. While it makes sense to look for such genuine uses in children's early productions (e.g., Bartsch and Wellman 1995), the practice when applied to parents' talk reduces the story-sharing interaction to a non-interactive third-person narration. This would be much like coding for what children would experience if they were listening to the story from an audio-book. A few exceptions in the literature do highlight the importance of coding for both the third-person (i.e., story characters) and the second-person (between the reader and the listener) references of mental state language. (Adrián et al. 2007; Taumoepeau and Ruffman 2006, 2008).

Among these, Taumoepeau and Ruffman (2006, 2008) have shown that parents' talk about 15-month-old children's own desires when describing pictures was the most consistent correlate of children's use of mental state language and their performance in emotion understanding tasks when they were 24-month-olds. In a follow up study with the same children at 33 months of age, it was found that the effectiveness of the child referent versus other referent categories had changed. Specifically, talk about others' (i.e., characters in the picture stimuli) cognitive states using "think" and "know" words was a more consistent predictor of children's emotion understanding and mental state use cross-sectionally. These two studies focused on the development of children's emotion understanding as opposed to their general theory of mind abilities of which emotion is one component. Nevertheless, the results are highly instructive. First, children benefited from different mental state categories at different ages (i.e., desire talk at 24 months, cognitive talk at 33 months). In addition, children benefited from different mental state talk that served to elucidate different perspectives (i.e., talk about the child at 24 months vs. talk about others at 33 months).

Adrián and colleagues (2007) have also coded separately for the cognitive verbs that mothers use in reference to the child or the mother as well as the language used in reference to story characters. This was a longitudinal study with Spanish speaking preschoolers that were tested 12 months apart. The results indicated that mental state talk in reference to the mother and the child were more consistent predictors of children's ToM abilities at Time 1 and Time 2. In contrast, mental state talk that referenced the story characters were only longitudinally related to children's ToM abilities.

These findings contribute to the SC argument where the results can be taken as evidencing parents' efforts to build common ground with their children in an interaction that is focused on the storybook. In everyday practice when parents read to their chil- 
dren, they monitor their children's understanding, emphasize and clarify important points through playful questions and extended discussion. This type of interaction, termed dialogic bookreading has been found to be an effective style that allows for children to enjoy and learn from books more effectively (for a meta-analysis see Mol et al. 2008), and has been used in training studies to train mothers to become more effective readers (Whitehurst et al. 1994). We argue that parents' interactions with their children about the story book is one of the critical strengths of this context. It facilitates parents' efforts to establish second-person common ground on third-persons' perspectives. Coding for adults' use of mental state language to express the desires, emotions, and thoughts of the characters eliminates the effort adults spend in getting the children to see the story as meaningful and interesting. When a mother asks a child "Where do you think the frog might be hiding?" or "You miss your friends when you don't see them for a long time, don't you?", she is effectively supporting the child's third-person perspective taking through second-person discourse. Studies that focus on both character-referenced and reader/listener-referenced talk should provide us with insight into whether children benefit from the scaffold of the second-person context in building their third-person socio-cognitive understanding. The few studies that have coded for such talk are instructive in this regard.

\section{The effect of conversation partner}

An overwhelming majority of research that looks at the effect of child-directed talk on children's ToM has exclusively included mothers as conversation partners. Some studies have recruited both mothers and fathers, however the proportion of fathers have remained woefully low (e.g., 3/74 in Taumoepeau and Ruffman 2006, 2008; 5/51 in Symons et al. 2005). One exception is a study by LaBounty and colleagues (2008), where they had both mothers and fathers talk about pictures depicting emotional situations to children at 3.5 years of age. The children were followed up a year later. It is important to note that the data for this study came from a larger longitudinal study where children with medium to high externalizing problems were oversampled. This may raise questions as to the generalizability of the findings. Nevertheless, the study reported intriguing results regarding the perspectival aspects of mothers' and fathers' discourse with their children, as well as how children benefited from these two different styles of discourse. Confirming reported findings that highlight the differences between mothers' and fathers' discourse, the study showed that mothers used significantly more emotion words and causal explanations of emotions as compared to fathers. Children seemed to benefit from mothers' emphasis on emotions as their emotion understanding was concurrently related to their mothers' emotion discourse. In contrast, fathers used more explanations of beliefs in their discourse. However, it was their causal explanatory language for desires and emotions that longitudinally predicted their children's ToM abilities even when children's IQ, ToM scores at Time 1 and mothers' mental state language use were controlled for.

These results may be interpreted within the SC approach such that children's co-constructive meaning making process is differentially affected by the different conversation partners. The TT theory has always argued for "crossover effects" of subtypes 
of mental state words for developing different components of ToM understanding. In other words, in the TT framework it is expected that children's FB understanding can be affected by the emotion or desire talk they are exposed to (Bartsch and Wellman 1995). However, the differential effect of different partners speaks more strongly to the effect of co-constructive processes because different conversation partners may show different patterns in scaffolding their children.

As stated earlier, this literature is dominated by research with mothers' language owing to the difficulty of recruiting fathers as active participants in research. However, such efforts are worthy undertakings if we aim to fully study the effect of child-directed language on their social-cognitive meaning making processes. It is our contention that we would be witnessing more variability of the kind reported in LaBounty et al. (2008) and have a deeper understanding of this relation.

\section{Mental state talk as dynamic scaffolding}

Mental state talk is one indicator of parents' perception of their children as mental agents that has been cleverly applied by the mind-mindedness approach. Mindmindedness research captures the extent to which mother's interact with their infants in ways that presuppose that their infants are mental agents. Elizabeth Meins and Charles Fernyhough have spearheaded the investigation of whether mind-mindedness in infancy scaffolds children's later ToM developments. To this end, their research program has included observations of infants and mothers in naturalistic interactions. Not only have they used comprehensive coding schemes for mothers' use of mental state language, but they have also assessed whether the appropriateness of mothers' mental state comments correlate with their children's later social-cognitive abilities (e.g. Kirk et al. 2015; Meins et al. 2003; Meins and Fernyhough 1999). This approach does not rely on the sheer frequency of mental state language used, instead, it investigates the concordance between the mothers' construal of their infants' desires, affect, and cognition and their infants' actual mental attitudes.

One of the important strengths of mind-mindedness research is that it focuses on the dynamic interplay of mental state attributions in infant-mother interactions. It also has the unique advantage of studying the early beginnings of such interplay as it scaffolds children's later ToM development. That said, this approach has not been effectively used with children who are preschool age or older in storytelling/picture book discourse. This is because appropriate versus inappropriate use of mental state language is no longer a category that will present meaningful individual variance in the talk of mothers, especially those from higher educational backgrounds as is most often the case for participants who volunteer for such studies. The forms of mindminded behavior should change depending on children's developmental level. For instance, in early infancy (e.g., 6 months) mind-mindedness is observed in mothers' use of appropriate mental state comments (i.e., comments that are consistent with the infant's actual mental state). In contrast, in the toddler years (e.g., 20 months) mother's attempts to make meaning of their children's incomprehensible verbalizations was found to correlate with later theory of mind (Meins and Fernyhough 1999). 
We argue that the way to study mind-mindedness using parents' mental state talk with preschoolers could proceed along three avenues, all of them largely unexplored. The first would investigate how the relationships between the frequency of mental state talk by category (e.g., perception, desire, emotion, belief) and ToM change for the same children. Very few of the longitudinal studies provide results that are relevant to understanding this possible change. Some studies have focused exclusively on the cognitive category (e.g., Adrián et al. 2007; Tompkins 2015) which makes it impossible to study the dynamic differences in the proportion of mental state categories relative to overall mental state language use. Others have not reported the change in subcategories but have worked with aggregate mental state talk scores (LaBounty et al. 2008). A notable exception is research by Taumoepeau and Ruffman $(2006,2008)$. In two papers, they track the effect of maternal talk on young children's emotion understanding from 15 months to 24 months to 33 months of age. One of the most intriguing result of this study is that while at 24 months children's emotion understanding was found to be related to their parents' desire talk at time 1, parents' desire talk at time 2 was negatively correlated to children's emotion understanding at 33-months of age. These results could be due to the fact that those parents who did not modify their ways of interacting with their child as their children grew older were adversely affecting their children's social-cognitive development. It is also possible to imagine an alternative scenario in which parents who implicitly knew their children had difficulty with social interactional content could be maintaining simpler talk to assist their children. Perhaps the only way to make sense of such negative correlations later in development is by working with the frequency of mental state language in the subcategories relative to the overall frequency of all mental state language (i.e., by working with proportions within the sub-categories) and tracking parents' individual variability of these proportions in relation to their children's social-cognitive gains over developmental time.

A second avenue would involve collecting language data in different contexts (e.g., storytelling and play) that are closely spaced in time. This would allow for the investigation of whether the categories of child-directed mental state talk that are related to children's ToM abilities vary by context. Parents' who are sensitive to the kind of scaffolding their children need in different contexts (e.g., picture book vs. reminiscing), may be expected to change their mental state talk patterns to accommodate their children's needs. If the nature of the relationships between mental state talk subcategories and ToM differ by context, this would shift the emphasis from mere exposure to mental state language to children's meaning making processes as situated in context with responsive social partners. To the best of our knowledge, a recent study by Devine and Hughes (2019) is the only one who sampled language in both storytelling and play contexts longitudinally from preschool children. However, in this study mother's use of mental state language was aggregated over contexts and so it does not provide us with clues about whether there was variability in the relations between the sub-categories of mental state words and children's ToM by context.

A final avenue of research could involve manipulating children's level of knowledge pertaining to the topic of discourse to see whether parents' talk about that topic changes accordingly. A natural way of accomplishing this would be to have parents read the same unfamiliar story to their children multiple times. The point would be to track 
whether and how parents modify their mental state talk as their child becomes more familiar with the book. This would allow us to see whether it is the sheer frequency of mental state language parents use at one time point or whether it is how they change their talk in relation to their children's level of knowledge about the book. This practice would actually prove ecologically valid, as new books are rarely read once to young children. Extensive research shows that children require multiple readings of stories to make meaning of the story plot and learn from them (e.g., Sénéchal 1997). In fact, interventions that target mothers and teachers of preschool children make explicit efforts to get adults to read books to children multiple times. These intervention programs are based on effective bookreading practices as naturally observed between parent-child dyads and explicitly instruct parents to alter their talk (i.e., their questions and comments) across multiple readings.

From a socio-cultural perspective mothers who are sensitive to their children's knowledge would alter talk their accordingly. This variation may not be observable in the sheer frequency of mental state use but the proportions of each subcategory relative to the total number of mental state usages. It is possible that mothers (and possibly not all mothers, but mind-minded mothers) may increase the proportion of cognitive language they use across readings, probing deeper into what Bruner (1986) has termed the "landscape of consciousness". It may be possible to identify mothers with certain patterns of mental state interaction with their child over time that scaffold their children's social cognitive understanding. Such research is missing at the moment but could be key to providing us with a better understanding of the role of social interaction in theory of mind development.

\section{Discussion}

In this paper, we aimed to discuss the implications of two constructivist frameworks on children's developing understanding of the mental world of agents. We argued that while these two approaches share in their endorsement of the child as an active meaning maker, they differ in terms their views on whether meaning making is as much a co-constructive process as it is a constructive process. In the SC approach, children's meaning making is embedded in social contexts that are fundamentally affected by the specifics of their experiential histories. As such, both mental state conceptions (e.g., belief, desire) as well as the ability to take and coordinate perspectives emerges out of the perspectival aspects of their interactions with social partners (Carpendale and Lewis 2004; Fernyhough 2008; Nelson 1996; Tomasello 2019).

According to the SC view, children can accomplish the challenging task of understanding mental states as guiding the behavior and attitudes of others by first learning to competently interact through meaningful social engagement. Further, as co-constructive partners, parents are responsive to their children's cognitive and social abilities. Consequently, they appropriate their ways of interacting with their children accordingly. As such our empirical investigations of the relationships between childdirected perspectival language (of which mental state talk is one component) should focus on the fine-grained analysis of interaction variables. These interaction variables include the nature of the language involved in perspectival talk (e.g., the categories 
of mental state talk included, non-mental state talk that implies perspective, etc.), as well as the pragmatic function of these mental state expressions (e.g., referencing third-persons vs. the speaker and the listener).

In addition, empirical work that aims to investigate whether SC or TT approaches better explain the relationships between child-directed perspectival talk and children's ToM should focus on change in such talk (e.g., across multiple contexts) and the relation of such change to ToM abilities both cross-sectionally but also longitudinally. To this end, comprehensive linguistic coding schemes should be employed such that the same parents and children are observed in multiple contexts longitudinally. This would allow researchers to investigate both cross-sectional differences to determine which specific aspects of talk support development in which specific contexts, but also whether these relationships change across developmental time.

To summarize, we have argued that the problem of emergence (i.e., the need for a foundationalism) and the inability to properly integrate culture into development constitute major theoretical limitations for the TT approach. However, our focus in the current paper has been to illustrate how theoretical differences between TT and SC have guided (and perhaps misguided) the design, coding, and analysis of empirical work. Further, we offer a prescriptive agenda for what it means to adopt a sociocultural approach to the empirical study of child-directed talk on children's ToM understanding. We have argued that carefully constructed research agendas that focus on variability (within speakers, across time, and between cultures) have the power to offer empirical evidence in favor of one theory over the other. Throughout this paper we have argued that research programs that take interaction in social situations as their unit of analysis (as opposed to mental state input) offer a vibrant empirical avenue that is theoretically motivated by a different set of ontological commitments. Such an approach should be able to take the plurality of folk-psychologies seriously and have the power to investigate what is regarded as universal from what is considered culture-specific.

Acknowledgements Preparation of this article was supported by a Grant to Hande Ilgaz from the Scientific and Technological Research Council of Turkey, (Grant No.: 217K169).

\section{References}

Adrián, J. E., Clemente, R. A., \& Villanueva, L. (2007). Mothers' use of cognitive state verbs in picturebook reading and the development of children's understanding of mind: A longitudinal study. Child Development, 78, 1052-1067. https://doi.org/10.1111/j.1467-8624.2007.01052.x.

Adrián, J. E., Clemente, R., Villanueva, L., \& Rieffe, C. (2005). Parent-child picture-book reading, mothers' mental state language and children's theory of mind. Journal of Child Language, 32(3), 673-686. https://doi.org/10.1017/S0305000905006963.

Allen, J. W., \& Bickhard, M. H. (2011). You can't get there from here: Foundationalism and development. Behavioral and Brain Sciences, 34, 124-125.

Allen, J. W. P., \& Bickhard, M. H. (2013). Stepping off the pendulum: Why only an action-based approach can transcend the nativist-empiricist debate. Cognitive Development, 28(2), 96-133. https://doi.org/ 10.1016/j.cogdev.2013.01.002.

Bartsch, K., \& Wellman, H. (1995). Children talk about the mind. New York: Oxford University Press.

Bickhard, M. H. (1980). Cognition, convention, and communication. Westport: Praeger Publishers. 
Bickhard, M. H. (1991). The import of Fodor's anti-constructivist argument. In L. Steffe (Ed.), Epistemological foundations of mathematical experience (pp. 14-25). New York: Springer.

Bickhard, M. H. (2008). Social ontology as convention. Topoi, 27(1-2), 139-149.

Bickhard, M. H. (2009a). Interactivism: A manifesto. New Ideas in Psychology, 27(1), 85-95. https://doi. org/10.1016/j.newideapsych.2008.05.001.

Bickhard, M. H. (2009b). The interactivist model. Synthese, 166(3), 547-591. https://doi.org/10.1007/ s11229-008-9375-x.

Bretherton, I., \& Beeghly, M. (1982). Talking about internal states: The acquisition of an explicit theory of mind. Developmental Psychology, 18(6), 906-921. https://doi.org/10.1037/0012-1649.18.6.906.

Bretherton, I., McNew, S., \& Beeghly-Smith, M. (1981). Early person knowledge as expressed in gestural and verbal communication: When do infants acquire a "theory of mind?". In M. E. Lamb \& L. R. Sherrod (Eds.), Infant social cognition. Hillsdale: Earlbaum.

Bruner, J. (1986). Actual minds, possible worlds. Cambridge: Harvard University Press.

Bruner, J. S. (1990). Acts of meaning (Vol. 3). Cambridge: Harvard University Press.

Carpendale, J. I., \& Carpendale, A. B. (2010). The development of pointing: From personal directedness to interpersonal direction. Human Development, 53(3), 110-126. https://doi.org/10.1159/000315168.

Carpendale, J., \& Lewis, C. (2004). Constructing an understanding of mind: The development of children's social understanding within social interaction. Behavioral and Brain Sciences, 27(1), 79-96. https:// doi.org/10.1017/S0140525X04000032.

Carpendale, J. I. M., \& Lewis, C. (2006). How children develop social understanding. Oxford: Blackwell.

Carpendale, J. I., \& Lewis, C. (2014). The development of children's understanding of social interaction. In The Routledge international handbook of young children's thinking and understanding, pp. 157-166. Abingdon: Routledge.

Chemero, A. (2009). Radical embodied cognitive science. Cambridge: MIT Press.

Christopher, J. C., \& Bickhard, M. H. (2007). Culture, self and identity: Interactivist contributions to a metatheory for cultural psychology. Culture and Psychology, 13, 259-295. https://doi.org/10.1177/ $1354067 X 07079881$.

Devine, R. T., \& Hughes, C. (2018). Family correlates of false belief understanding in early childhood: A metaanalysis. Child Development, 89, 971-987. https://doi.org/10.1111/cdev.12682.

Devine, R. T., \& Hughes, C. (2019). Let's talk: Parents' mental talk (not mind-mindedness or mindreading capacity) predicts children's false belief understanding. Child Development, 90(4), 1236-1253. https:// doi.org/10.1111/cdev.12990.

Ensor, R., Devine, R. T., Marks, A., \& Hughes, C. (2014). Mothers' cognitive references to 2-year-olds predict theory of mind at ages 6 and 10. Child Development, 85, 1222-1235.

Fernyhough, C. (2008). Getting Vygotskian about theory of mind: Mediation, dialogue, and the development of social understanding. Developmental Review, 28(2), 225-262. https://doi.org/10.1016/j.dr.2007.03. 001.

Fivush, R. (2019). Sociocultural developmental approaches to autobiographical memory. Applied Cognitive Psychology, 33(4), 489-497. https://doi.org/10.1002/acp.3512.

Fodor, J. A. (1975). The language of thought. New York City: Thomas Y. Crowell.

Gallagher, S. (2001). The practice of mind: Theory, simulation, or interaction? Journal of Consciousness Studies, 8, 83-107.

Göksel, A., \& Kerslake, C. (2005). Turkish: A comprehensive grammar. London: Routledge.

Gopnik, A. (1996). The scientist as child. Philosophy of Science, 63, 485-514.

Gopnik, A. (2003). The theory theory as an alternative to the innateness hypothesis. In L. Antony \& N. Hornstein (Eds.), Chomsky and his critics. New York: Basil Blackwell.

Gopnik, A., \& Wellman, H. M. (1992). Why the child's theory of mind really is a theory. Mind and Language, 7, 145-171.

Hutto, D. D., \& Myin, E. (2012). Radicalizing enactivism: Basic minds without content. Cambridge: MIT Press.

Kirk, E., Pine, K., Wheatley, L., Howlett, N., Schulz, J., \& Fletcher, B. C. (2015). A longitudinal investigation of the relationship between maternal mind-mindedness and theory of mind. British Journal of Developmental Psychology, 33, 434-445. https://doi.org/10.1111/bjdp.12104.

LaBounty, J., Wellman, H. M., Olson, S., Lagattuta, K., \& Liu, D. (2008). Mothers' and fathers' use of internal state talk with their young children. Social Development, 17, 757-775. https://doi.org/10. 1111/j.1467-9507.2007.00450.x. 
Laible, D. J. (2004). Mother-child discourse in two contexts: Links with child temperament, attachment security, and socioemotional competence. Developmental Psychology, 40, 979-992. https://doi.org/ 10.1037/0012-1649.40.6.979.

Laible, D. J. (2011). Does it matter if preschool children and mothers discuss positive vs. negative events during remi- niscing? Links with mother-reported attachment, family emotional climate, and socioemotional development. Social Development, 20, 394-412. https://doi.org/10.1111/j.1467-9507.2010. 00584.x.

Lillard, A. (1998). Ethnopsychologies: Cultural variations in theories of mind. Psychological Bulletin, 123(1), 3. https://doi.org/10.1037/0033-2909.123.1.3.

Liu, D., Wellman, H. M., Tardif, T., \& Sabbagh, M. A. (2008). Theory of mind development in Chinese children: A meta-analysis of false-belief understanding across cultures and languages. Developmental Psychology, 44, 523-531. https://doi.org/10.1037/0012-1649.44.2.523.

Meins, E., \& Fernyhough, C. (1999). Linguistic acquisitional style and mentalising development: The role of maternal mind-mindedness. Cognitive Development, 14, 363-380. https://doi.org/10.1016/s08852014(99)00010-6.

Meins, E., Fernyhough, C., Arnott, B., Leekam, S. R., \& Rosnay, M. (2013). Mind mindedness and theory of mind: Mediating roles of language and perspectival symbolic play. Child Development, 84(5), 1777-1790. https://doi.org/10.1111/cdev.12061.

Meins, E., Fernyhough, C., Wainwright, R., Clark-Carter, D., Das Gupta, M., Fradley, E., et al. (2003). Pathways to understanding mind: Construct validity and predictive validity of maternal mind-mindedness. Child Development, 74, 1194-1211. https://doi.org/10.1111/1467-8624.00601.

Meins, E., Fernyhough, C., Wainwright, R., Das Gupta, M., Fradley, E., \& Tuckey, M. (2002). Maternal mind-mindedness and attachment security as predictors of theory of mind understanding. Child Development, 73(6), 1715-1726. https://doi.org/10.1111/1467-8624.00501.

Melzi, G., Schick, A. R., \& Kennedy, J. L. (2011). Narrative elaboration and participation: Two dimensions of maternal elicitation style. Child Development, 82(4), 1282-1296. https://doi.org/10.1111/j.14678624.2011.01600.x.

Miller, S. A. (2016). Parenting and theory of mind. Oxford: Oxford University Press.

Miller, P. J., Sandel, T. L., Liang, C. H., \& Fung, H. (2001). Narrating transgressions in Longwood: The discourses, meanings, and paradoxes of an American socializing practice. Ethos, 29(2), 159-186. https://doi.org/10.1525/eth.2001.29.2.159.

Mirski, R., \& Gut, A. (2018). Action-based versus cognitivist perspectives on socio-cognitive development: Culture, language and social experience within the two paradigms. Synthese. https://doi.org/10.1007/ s11229-018-01976-y.

Mol, S. E., Bus, A. G., Jong, M. T., \& Smeets, D. J. (2008). Added value of dialogic parent-child book readings: A meta-analysis. Early Education and Development, 19, 7-26. https://doi.org/10.1080/ 10409280701838603.

Nelson, K. (1996). Language in cognitive development: The emergence of the mediated mind. New York: Cambridge University Press.

Nelson, K. (2005). Language pathways to the community of minds. In J. W. Astington \& J. Baird (Eds.), Why language matters to theory of mind (pp. 26-49). New York: Oxford University Press.

Nelson, K. (2007). Young minds in social worlds: Experience, meaning, and memory. Cambridge: Harvard University Press.

Nelson, K. (2009). Narrative practices and folk psychology: A perspective from developmental psychology. Journal of Consciousness Studies, 16(6-7), 69-93.

Nelson, K. (2010). Developmental narratives of the experiencing child. Child Development Perspectives, 4, 42-47. https://doi.org/10.1111/j.1750-8606.2009.00116.x.

Nelson, K., Plesa, D., \& Henseler, S. (1998). Children's theory of mind: An experiential interpretation. Human Development, 41(1), 7-29.

Nelson, K., Skwerer, D. P., Goldman, S., Henseler, S., Presler, N., \& Walkenfeld, F. F. (2003). Entering a community of minds: An experiential approach to 'theory of mind'. Human Development, 46(1), 24-46. https://doi.org/10.1159/000067779.

Ontai, L. L., \& Thompson, R. A. (2008). Attachment, parent-child discourse, and theory-of-mind development. Social Development, 17, 47-60. https://doi.org/10.1111/j.1467-9507.2007.00414.x.

Peterson, C. C., \& Siegal, M. (2000). Insights into theory of mind from deafness and autism. Mind and Language, 15(1), 123-145. https://doi.org/10.1111/1468-0017.00126. 
Peterson, C., \& Slaughter, V. (2003). Opening windows into the mind: Mothers' preferences for mental state explanations and children's theory of mind. Cognitive Development, 18, 399-429. https://doi. org/10.1016/S0885-2014(03)00041-8.

Piaget, J. (1954). The construction of reality in the child. (M. Cook, Trans.). New York: Basic Books. (Original work published 1937).

Racine, T. P., Carpendale, J. I. M., \& Turnbull, W. (2007). Parent-child talk and children's understanding of beliefs and emotions. Cognition and Emotion, 21, 480-494. https://doi.org/10.1080/ 02699930600717599.

Reddy, V. (2007). Getting back to the rough ground: deception and 'social living'. Philosophical Transactions of the Royal Society B: Biological Sciences, 362, 621-637.

Ruffman, T., Slade, L., \& Crowe, E. (2002). The relation between children's and mothers' mental state language and theory-of-mind understanding. Child Development, 73(3), 734-751. https://doi.org/10. $1111 / 1467-8624.00435$.

Selcuk, B., Brink, K. A., Ekerim, M., \& Wellman, H. M. (2018). Sequence of theory-of-mind acquisition in Turkish children from diverse social backgrounds. Infant and Child Development, 27(4), e2098. https://doi.org/10.1002/icd.2098.

Sénéchal, M. (1997). The differential effect of storybook reading on preschoolers' acquisition of expressive and receptive vocabulary. Journal of Child Language, 24(1), 123-138. https://doi.org/10.1017/ S0305000996003005.

Shahaeian, A., Peterson, C. C., Slaughter, V., \& Wellman, H. M. (2011). Culture and the sequence of steps in theory of mind development. Developmental Psychology, 47(5), 1239. https://doi.org/10.1037/ a0023899.

Shatz, M., Wellman, H. M., \& Silber, S. (1983). The acquisition of mental verbs: A systematic investigation of the first reference to mental state. Cognition, 14(3), 301-321. https://doi.org/10.1016/00100277(83)90008-2.

Slaughter, V., Peterson, C. C., \& Mackintosh, E. (2007). Mind what mother says: Narrative input and theory of mind in typical children and those on the autism spectrum. Child Development, 78, 839-858. https:// doi.org/10.1111/j.1467-8624.2007.01036.x.

Symons, D. K., Fossum, K. L. M., \& Collins, T. B. K. (2006). A longitudinal study of belief and desire state discourse during mother-child play and later false belief understanding. Social Development, 15, 676-692. https://doi.org/10.1111/j.1467-9507.2006.00364.x.

Symons, D. K., Peterson, C. C., Slaughter, V., Roche, J., \& Doyle, E. (2005). Theory of mind and mental state discourse during book reading and story-telling tasks. British Journal of Developmental Psychology, 23, 81-102. https://doi.org/10.1348/026151004X21080.

Taumoepeau, M., \& Ruffman, T. (2006). Mother and infant talk about mental states relates to desire language and emotion understanding. Child Development, 77, 465-481. https://doi.org/10.1111/j.1467-8624. 2006.00882.x.

Taumoepeau, M., \& Ruffman, T. (2008). Stepping stones to others' minds: Maternal talk relates to child mental state language and emotion understanding at 15, 24, and 33 months. Child Development, 79, 284-302. https://doi.org/10.1111/j.1467-8624.2007.01126.x.

Tomasello, M. (1999). The cultural origins of human cognition. Cambridge: Harvard University Press.

Tomasello, M. (2019). Becoming human: A theory of ontogeny. Cambridge: Harvard University Press.

Tomasello, M., Carpenter, M., \& Liszkowski, U. (2007). A new look at infant pointing. Child Development, 78(3), 705-722. https://doi.org/10.1111/j.1467-8624.2007.01025.x.

Tompkins, V. (2015). Mothers' cognitive state talk during shared reading and children's later false belief understanding. Cognitive Development, 36, 40-51. https://doi.org/10.1016/j.cogdev.2015.08.004.

Tompkins, V., Benigno, J. P., Lee, B. K., \& Wright, B. M. (2018). The relation between parents' mental state talk and children's social understanding: A meta-analysis. Social Development, 27, 1-14. https:// doi.org/10.1111/sode.12280.

Vygotsky, L. S. (1997). Genesis of higher mental functions (M. J. Hall, Trans.). In R. W. Rieber (Ed.) The collected works of L. S. Vygotsky: The history of the development of higher mental functions, vol. 4, pp. 97-119. New York: Plenum Press. (First published in 1931).

Wellman, H. (1990). The child's theory of mind. Cambridge: MIT Press.

Wellman, H. M. (2014). Making minds: How theory of mind develops. New York: Oxford University Press.

Wellman, H. M., Fang, F., Liu, D., Zhu, L., \& Liu, G. (2006). Scaling of theory-of-mind understandings in Chinese children. Psychological Science, 17(12), 1075-1081. https://doi.org/10.1111/j.1467-9280. 2006.01830.x. 
Wellman, H. M., \& Liu, D. (2004). Scaling of theory-of-mind tasks. Child Development, 75, 523-541. https://doi.org/10.1111/j.1467-8624.2004.00691.x.

Whitehurst, G. J., Arnold, D. S., Epstein, J. N., Angell, A. L., Smith, M., \& Fischel, J. E. (1994). A picture book reading intervention in day care and home for children from low-income families. Developmental Psychology, 30(5), 679. https://doi.org/10.1037/0022-0663.86.4.542.

Ziemke, T. (2003). What's that thing called embodiment? Proceedings of the Annual Meeting of the Cognitive Science Society, 25, 1305-1310.

Publisher's Note Springer Nature remains neutral with regard to jurisdictional claims in published maps and institutional affiliations. 